\title{
On the Cosmology of Electromagnetic Wave Energy in Expanding Universes
}

\author{
Hans-Jörg Fahr ${ }^{1 *}$ and Michael Heyl ${ }^{2}$ \\ ${ }^{1}$ Argelander Institut für Astronomie, Universität Bonn, 53121 Bonn, Germany \\ ${ }^{2}$ Space Administration, German Aerospace Center, 53229 Bonn, Germany \\ Email: hfahr@astro.uni-bonn.de
}

\begin{abstract}
Modern cosmology considers several different ingredients entering the energy-momentum tensor of the GTR-field equations, amongst them normal baryonic matter, dark matter, dark energy and photons. The photons are usually taken as negligibly influencing the present-day expansion dynamics because they are seen as permanently loosing energy due to being cosmologically redshifted. In an earlier study we have discussed why this view is questionable and why freely propagating photons, while being transported in an expanding universe, do not change their energy. In this paper, instead of using a cosmic photon view, we treat the cosmic electromagnetic radiation field as a system of monodirectional wave modes. Single photons moving with the velocity of light can not be described as sources of gravity, raising the question how cosmic photons hence may contribute to the cosmic gravity field. Here we conclude that photons or the associated electromagnetic waves can only be described as gravity sources, if they constitute a form of localized standing energy. To represent localized energy, electromagnetic waves in an appropriate manner have to interfere with their counter-propagating waves of an appropriate phase shift $\pi$ to produce standing waves.

We describe the energy distribution of monochromatic standing waves and consider these monodirectional, monochromatic wave modes as undamped wave modes freely extending over the dimension of the universe. We show that they keep a constant mode energy despite the cosmic expansion connected with the shift of their wavelengths proportional to the cosmic scale $S$. With these considerations we obtain an expression for the energy density of standing electromagnetic waves and show that their wave energy density scales with $S^{-3}$, instead like $S^{-4}$ as expected for the cosmic radiation energy density by the present-day cosmology. We conclude that with our present result we confirm an earlier study carried out on the basis of freely propagating single photons, since on both ways we find that the energy density of cosmic electromagnetic radiation scales identically to the scaling of the cosmic matter density, namely according to $S^{-3}$.
\end{abstract}

Keywords: Cosmic background radiation - dark matter - theory

\section{Introduction}

At least with some intellectual profit, one can consider universes with an energy content constituted by nothing else but electromagnetic radiation, or to say it in other words, by photons only. Assuming that there exists a universe without any matter content, i.e. without dark or normal particles with rest masses, and without dark energy as the standard ingredient of modern cosmology nowadays, then one would be left with a universe that only contains photons. This conceptual view is not even useless or irrational, since imagining that at the beginning of cosmic evolution we would have had a completely balanced mixture of particles and anti-particles (koino-matter, as in accordance with the baryon conservation law) which then have to undergo pair-annihilation below the pair-production temperature limit, this then should have left for the rest of the cosmic times a universe with nothing else but annihilation photons. For that scenario then it substantially counts how cosmic photons freely propagating in the universe behave under the dynamics of the expanding universe, i.e. how are these photons redistributed in cosmic configuration space and in frequency space at the expansion of the universe?

On the other hand, the expansion dynamics of the universe is determined by the energy-momentum tensor $T_{i k}$ which, in the special case that is faced here, is constituted exclusively by photonic energy representations. These photons, though not having rest masses, nevertheless represent a form of energy $E_{\nu}=h \nu$ and a dynamical mass $m_{\nu}=\left(h \nu / c^{2}\right)$ and hence, in the sense of the GTR, have to somehow curve 
the cosmic 4d-spacetime geometry through their corresponding ingredients in the GTR energy-momentum tensor $T_{i k}$. Though pure photon universes appear to be something very special and exotic, perhaps even utopic, they fully justify a thorough theoretical investigation. This in fact is the reason why these types of universes have nevertheless already been discussed in the cosmologic literature at many places (see e.g. [1], [2], [3], [4], [5]). Usually in these studies it is presented as the result that cosmic photons embedded in the spacetime geometry of the expanding universe are subject to a permanent redshift, called the "cosmological redshift" by which the wavelength $\lambda$ of each cosmic photon grows proportional to the scale size $S$ of the universe according to the scaling law $\lambda / \lambda_{0}=S / S_{0}$. This redshift result represents the GTR generalization of the STR redshift explanation which first was the basis of the standard explanation of galactic redshifts at the time of Hubble's famous detection of the expanding universe ([6]) and thereafter, but had to be given up for non-linear redshifts larger than $z \geq 0.2$.

The question of the redshifting of cosmic photons only received some newly perspected considerations by e.g. [7], [8], [9], [10], [11]. Only most recently then a paper was published ([12]) where the nowadays well accepted canonical GTR interpretation of the cosmic photon redshift was rediscussed and at least rejected in parts. The main point therein was that cosmic photons in their own proper system, while propagating on cosmic light-geodetics, do not experience the touch of the expanding universe and do not change their proper energy, rather the cosmological energy loss is only registered later, when these photons at some later cosmic times are measured, i.e. spectrally analyzed. Treating cosmic photons as particles this would imply that the energy density of such cosmic photons would decrease as $S^{-3}$ in contrast to the prediction of the standard cosmology saying that it decreases like $S^{-4}$. As shown by [12] this result has substantial consequences for the contribution of photons to the cosmic energy-momentum tensor in the present days of the universe. In the following article we want to analyse deeper whether these most relevant implications are only due to the particle view which was applied to photons by [12], or whether these results also find their support when taking as alternative a wave-view to the cosmic CMB photons.

\section{Energy Representation based on Electromagnetic Wave Modes}

First we want to put the question here whether or not photons with no rest mass can at all be sources of genuine gravitational fields and not only can react to rest-mass induced, existing gravitational fields. We agree and acknowledge the fact that photons themselves are affected by the latter gravitational fields, like those e.g. of stellar masses, leading to the well known photon deflections predicted by Einstein or to Moessbauer's photon energy increases in gravitational fields (see [13]). But what will happen in case that the stellar mass is constituted by an ambimatter star (i.e. equal numbers of baryons and anti-baryons) when these baryons and antibaryons - completely annihilate into photons, i.e. convert baryonic into photonic forms of energy? Do photons attract photons? Do they constitute the same gravitational field as did the baryons before? Since the annihilation process conserves the energy, this would mean in case the originating photons would be impeded in leaving the star, if e.g. it were a "black star", would this "black star" then still produce the same gravitational field and thus still enforce a single photon outside of the Schwartzschild sphere to experience the same deflection as before annihilation?

If, for instance, two single photons would come close enough to each other, would they gravitationally interact with each other due to their mere energy representations? And if at all, how would they do it? Would it lead to mutual deflections of the respective photons, kicking the two photons into new propagation directions? If this latter would be the case, then on the long run cosmic photons could not be considered to be "freely" propagating objects, but objects gravitationally mutually interacting with each other. On the other hand, photons are objects propagating with the velocity of light. If they would create at their light-like propagation gravitational fields by emitting gravitational waves, then these fields would appear strongly asymmetric and due to the resulting action aberration would mean that photons can only gravitationally interact with photons propagating just opposite to them, because the action angle for particles moving with a velocity $v=c$ (i.e. photons) becomes 180 degrees. (see [14]).

Photons, in order to mutually change their energy by mutual gravitational interactions should, however, approach each other to distances smaller than: 


$$
\frac{G}{r} \cdot\left(\frac{h \nu}{c^{2}}\right)^{2} \approx h \nu
$$

leading to the requirement

$$
r \approx G(h \nu) / c^{4}
$$

which e.g. for a photon with energy $h \nu=1 \mathrm{KeV}$ leads to the requirement: $r(1 \mathrm{KeV}) \approx 10^{-58} \mathrm{~cm}$.

Since these are rather exotic requirements, one could, in order to perhaps gain more credit for these considerations, look at these things with an alternative view: Photons can be easily handled with the standards of gravitation theory, if they can be considered not as light-velocity-fast moving energy quantities, but as standing energy representations in 3D-space. In this respect we would like to remind the reader that photons are representations of electromagnetic waves propagating with the velocity of light. Only when two of these electromagnetic waves with identical wavelength and the appropriate phase shift are interfering with each other, then so-called standing waves will result from that and the non-propagating relevant field energy is deposited in the consecutive bulges of this interference pattern. In this form of a locally standing energy, the waves could easily be considered as standard sources of gravitational fields. Completely standing waves, however, can only occur, if the interfering counter-propagating waves allover have the correct phase shift of $\chi=\pi$. Within a closed cavity this can be achieved, if waves are reflected at the cavity borders with a phase change of $\Delta \chi=\pi$ (i.e. total reflection at the border!). Under such conditions counter-propagating waves would store and localize their energy in the bulges of the wave interference patterns, i.e. the wave energy distribution would be stationary and spatially discretized.

In a homogeneous universe obeying the "cosmological principle", discretized energy depositions should, however, not be allowed (i.e. no priorities to special 3D-places!), and thus cosmic radiation fields should have homogeneously distributed energy knots and bulges in order to guarantee a cosmologically relevant homogeneous energy deposition. This means energy bulges in their modes along the direction of the wave propagation should be equally distributed, established by a homogeneous phase distribution of such waves (i.e. all phases $\chi$ of such a wave mode must be equally probable!). To take care of this "cosmologically" required fact one needs to introduce as energy density the integral of the spectral energy distribution $u_{\lambda}(\chi)$ over all phases $\chi$ with equal phase probability. This then leads to the result :

$$
\bar{u}_{\lambda}=\left\langle E(\lambda)^{2}\right\rangle_{\chi}=(4 / 3) E^{2}(\lambda)
$$

In addition, the magnetic energy contribution to the electric energy part does not represent an additional problem concerning phase distributions. This is because it is shown in text book literature on electromagnetic vacuum waves that the linearly polarized electromagnetic wave with $k=k_{z} ; E=$ $E_{y} ; B=B_{x}$, to fulfill Maxwell's equations, has no phase shift for instance between the $E_{y}$-component and the $B_{x}$-component of the same electromagnetic wave mode.

On the other hand, to require total reflection of electromagnetic wave modes at the border of the cosmic cavity system would force to also consider the action of a wave pressure on such border surfaces. It may perhaps even simply be a request of the cosmological principle that due to the required symmetry in the existing modes for each mode naturally a corresponding counter-mode should be existing whatever is its generator. One could as well subdivide the universe into many sub-universes repeating each other in all dimensions. Hence considering the border of such a sub-universe as reflector of the considered wave modes, one would have a corresponding reflection of identical modes on the outside of this sub-universe. Under these conditions one can see that the pressure of the reflected wave modes cancels and does not need to be taken into account.

Furthermore it also would not fulfill and correspond to the cosmological principle to only consider linearly polarized $E_{y}$-waves prefering the y-direction. This means that as the relevant cosmic electromagnetic waves one should expect to have the $E_{y}$-waves compensated by an adequate appearance of $E_{x}$-waves to not allow for any preferred polarization direction. This means that in connection with the above mentioned energy density for the linearly polarized mode for the cosmic system one should expect to get a factor "2" yielding the complete energy density by

$$
\bar{u}_{\lambda}=\left\langle E_{x}(\lambda)^{2}\right\rangle_{\chi}+\left\langle E_{y}(\lambda)^{2}\right\rangle_{\chi}=(8 / 3) E^{2}(\lambda)
$$




\section{Cosmic Redshift of Wave Modes}

Now the additional question may be put, how much the monochromatic wave would recognize that the reflecting border is moving apart with the Hubble expansion of the universe? Since the waves propagate in the co-moving cosmological rest frame, one can conclude that wavelengths due to the interaction with expanding borders of the propagation box are subject to the Hubble expansion. Over one wavelength $\lambda$, this Hubble expansion leads to a specific wavelength expansion given by

$$
\dot{\lambda}=H \cdot \lambda
$$

This means that the relative wavelength change per time is given by

$$
\dot{\lambda} / \lambda=H(t)
$$

correspondingly leading to the following wavelength $\lambda(t)$ as function of cosmic time:

$$
\lambda(t)=\lambda\left(t_{0}\right) \exp \left[\int_{t_{0}}^{t} H(t) d t\right]
$$

This also means that the wavelength change per wavelength would be $\frac{d \lambda}{d t}=\frac{\lambda}{c} \dot{\lambda}=H \frac{\lambda^{2}}{c}$ meaning that during one crossover through the dimension $S$ of the universe this would result to a wavelength change per crossover time by

$$
\Delta \lambda_{S}=\frac{S}{c} H \lambda=S H \frac{\lambda}{c}
$$

The change of the mode wavelength derived with the upper formula (8) means that, taken this together with the result of the section before, not only the power of the mode $E_{0}^{2}(\lambda)$ decreases with the cosmic scale according to $(1 / S(t))$, but also this reduced power at a later cosmic time is transported to another, namely larger wavelength $\lambda(t) \geq \lambda\left(t_{0}\right)$ which in view of the upper result and the fact that the Hubble parameter is defined by $H(t)=\dot{S}(t) / S(t)$ can simply be given by the following formula

$$
\begin{aligned}
\lambda(t) & =\lambda\left(t_{0}\right) \exp \left[\int_{t_{0}}^{t} \frac{\dot{S}(t)}{S(t)} d t\right] \\
& =\lambda\left(t_{0}\right) \exp \left[\int_{t_{0}}^{t} \frac{d \ln (S(t))}{d t} d t\right]=\lambda\left(t_{0}\right) \frac{S(t)}{S\left(t_{0}\right)}
\end{aligned}
$$

This result is consistent with the classic present-day interpretation of cosmic photon redshifts, however, its derivation and its physical interpretation is different. The change of the mode wavelength derived with the upper formula then means that, taken this together with the result of the section before, not only the power of the mode $E_{0}^{2}(\lambda)$ decreases with the cosmic scale according to $(1 / S(t))$, but also this reduced power at a later cosmic time is replaced to an other, namely larger wavelength $\lambda(t)=\lambda\left(t_{0}\right) \cdot\left[S(t) / S\left(t_{0}\right)\right]$, implying a corresponding spectral energy shift in an existing cosmic radiation field.

\section{The Energy of Cosmic Electromagnetic Wave Modes}

In this section we consider cosmic photons represented by mono-directional, finite 1-D wave modes extended over a total cosmic length extension of $S$, equal to the scale of the universe. We assume a one-dimensional $(+\mathrm{z})$-directed, y-linear polarized, electromagnetic, mono-chromatic wave with an electric field given by

$$
E(z, \lambda)=E_{y}(\lambda) \cos [z / \lambda]
$$

where $E_{y}(\lambda)$ denotes the $E$-field amplitude of the mode with wavelength $\lambda$, propagating into the z-direction over a monodirectional dimension $S$ of the universe, till it is reflected at the outer $(+z)$-border of the universe, producing via phase change by $\pi$ a wave knot there and a counter-propagating antiwave in the 
opposite $(-z)$-direction. This reflected wave interferes with the primary wave to produce a standing wave between the two opposite $(+z /-z)$ - borders of the universe.

The electromagnetic energy density of this wave at a location $z$ is given by:

$$
u_{y}(\lambda)=E_{y}^{2}(\lambda) \cos ^{2}[z / \lambda]
$$

Let the number $n$ of consecutive wavelengths $\lambda$ fitting into the length $S$ be:

$$
n=\frac{S}{\lambda}
$$

Then the electromagnetic energy $\Xi_{y}(\lambda)$ contained within the full extension $S$ of this standing wave (i.e. cosmic energy column density of the wave mode) is given by:

$$
\begin{aligned}
\Xi_{y}(\lambda) & =E_{y}^{2} \int_{0}^{S=n \lambda} \cos ^{2}[z / \lambda] d z \\
& =n E_{y}^{2} \int_{0}^{\lambda} \cos ^{2}[z / \lambda] d z=n \frac{1}{2} E_{y}^{2} \lambda=\frac{1}{2} E_{y}^{2} S
\end{aligned}
$$

Assuming that this quantity, due to loss-free radiation propagation (i.e neither cosmic radiation absorbers nor emitters since we only consider freely propagating CMB radiation here), is conserved in the expanding universe, $\Xi_{y}(\lambda)=$ const., then would mean that the following scaling has to be expected

$$
E_{y}^{2}(\lambda) / E_{y 0}^{2}(\lambda)=S_{0} / S
$$

with $S(t)$ and $S_{0}\left(t_{0}\right)$ the cosmic scale parameters at cosmic times $t$ and $t_{0}$.

Thinking now of an isotropic radiation field of such monochromatic $\lambda$ waves with $E(x, \lambda)=E(y, \lambda)=$ $E(z, \lambda)$ does then mean that the total energy column density $\Xi(\lambda)$ of such a 3D-mode thus is given by the superposition of the three spatial modes and thus given by

$$
\Xi(\lambda)=\sum_{i=1}^{3} \Xi_{i}(\lambda)=\frac{1}{2} S \cdot \sum_{i=1}^{3} E_{i 0}^{2}(\lambda)
$$

which thus, perhaps surprisingly, means that also for an isotropic radiation field the associated monochromatic wave energy density $u(\lambda)$ in that case drops off according to

$$
u(\lambda) / u_{0}=E^{2}(\lambda) / E_{0}^{2}(\lambda)=S_{0} / S
$$

This result must be valid for any wavelength $\lambda$ and one could think that the total electromagnetic energy density $u_{e m}$ of all wave modes with arbitrary wavelength $\lambda$ would be:

$$
\begin{aligned}
u_{\mathrm{em}}(S) & =\sum_{\lambda} u(\lambda)=\sum_{\lambda} E^{2}(\lambda) \\
& =\frac{S_{0}}{S} \sum_{\lambda} E_{0}^{2}(\lambda)=\frac{S_{0}}{S} \int_{0}^{\infty} \frac{d E_{0}^{2}(\lambda)}{d \lambda} d \lambda
\end{aligned}
$$

with a spectral wavelength distribution $d E_{0}^{2}(\lambda) / d \lambda$ described e.g. by a Planck curve for a given temperature $T_{0}$ at a scale parameter $S_{0}$.

At this point we must remind the reader that the above derivation is correct, though the total energy of each wave mode with wavelength $\lambda$ is argued as pointed out to be conserved. However, it also turned out in section 3 that the wavelength spread $d \lambda$, like $\lambda$ itself, increases proportional to $S$. That means, while the averaged energy density $u(\lambda)$ of a wave mode itself decreases with $1 / S$ and at the same time its wavelength increases proportional to $S$ during the expansion of the universe, the total amount of energy of this cosmic wave mode (= cosmic energy column density $\Xi(\lambda)$ ) remains unchanged, but being distributed over an enlarged wavelength interval placed at an enlarged wavelength $\lambda$. 
Or saying it in a different form: All the wave modes keep their total amount of energy constant despite the change of their associated wavelengths, and thus the total electromagnetic energy represented by them is constant within the increasing cosmic volume. Since the volume increases with $S^{3}$, this then evidently means, that the total electromagnetic energy density $\left[u_{e m}\right]$ must scale with $S^{-3}$ : (the latter quantity must be distinguished from $u_{e m}(S)$ in Eq. (17)!)

$$
\left[u_{\mathrm{em}}(S)\right]=\left[u_{\mathrm{em}, 0}\right] \frac{S_{0}^{3}}{S^{3}}
$$

Here the factor $\left[u_{\mathrm{em}, 0}\right]$ represents the total electromagnetic energy density at the reference time $t_{0}=$ $t_{r}$, or for the scale $S_{r}$, of recombination,

$$
\left[u_{\mathrm{em}, 0}\right]=\left[u_{\mathrm{em}}\left(S_{r}\right)\right]=\int_{0}^{\infty} \frac{d E_{0}^{2}(\lambda)}{d \lambda} d \lambda
$$

where the integral is carried out over the associated Planck distribution at recombination time with temperature $T_{r} \approx 3000 K$. This integral as is well known results in

$$
\left[u_{\mathrm{em}, 0}\right]=\left[u_{\mathrm{em}}\left(S_{r}\right)\right]=\sigma_{\mathrm{sb}} T_{r}^{4}
$$

with $\sigma_{\mathrm{sb}}$ being the so-called Stefan-Boltzmann constant.

Using Eq. (18) one can then state that the final result of the above considerations culminates in stating that the electromagnetic energy density in an expanding universe must follow the law:

$$
\left[u_{\mathrm{em}}(S)\right]=\frac{S_{0}^{3}}{S^{3}} \sigma_{\mathrm{sb}} T_{r}^{4}
$$

Interestingly enough, this result is identical with the earlier finding by Fahr \& Heyl (2017) where the free propagation of light from the recombination period onwards has been investigated on the basis of a "particle point of view", i.e. a cosmic photon view. So this makes evident that we obtain full conciliation between cosmic photon and wavemode views.

\section{Conclusions}

The above presented wavemode model is able to combine mode energy conservation with the empirical fact of cosmological photon redshift by delivering the following results:

- it solves the always as "mystic" considered problem as to where the energy of redshifted photons has gone: namely No-where!, but this energy is still and always there. The whole energy is just redistributed along an increasingly elongated wave mode

- it is conciliatory with the generally accepted assumption that the wavelengths of freely propagating photons in an expanding universe increase with cosmic expansion scale $S$ as shown in section (3).

- it is conciliatory with the general expectation that the energy density of CMB photons scales with $1 / S^{4}$, since this is what in fact the observer with a present-day spectrometer sees.

- it also confirms - now regarding wavemodes from a photon view - the recently published idea of [12] that photons in their peculiar photon rest frame do not suffer from the expansion of the universe.

- and it supports the idea in the above mentioned paper that the total electromagnetic energy density at present times, which is roughly by a factor $z=1100$ higher than in the standard model $\left(S^{-3}\right.$ instead of $S^{-4}$ ), is comparable with the energy density of matter and can therefore explain a certain amount of "Dark Matter".

Finally, it is worth to mention that the concept of an "energy conserving redshift" can also be applied to photons when regarded as finite wave packets with length $L=c \tau$. Here, $\tau$ is equal to $(1 / \nu)$ or the lifetime e.g. of an atomic electron transition. Also in the wave packet-view the length of the wave packet $L=n \lambda$ and thus $\lambda$ increases according to $S / S_{0}$ while the total energy of the wave packet remains constant and is just distributed along the "stretched" finite wave mode. 


\section{References}

1. M. Berry, Kosmologie und Gravitation. Teubner, Stuttgart, 1990

2. C.W. Misner, K.S. Thorne and J.A. Wheeler, Gravitation. Freeman, San Francisco, 1973

3. J.A. Peacock, Cosmological Physics. Cambridge/MA: Cambridge University Press, 1999

4. R. Sexl and H. Urbantke, Relativität, Gruppen, Teilchen. Springer, Wien, 1987

5. H.F.M Goenner, Einführung in die Spezielle und Allgemeine Relativitätstheorie. Spektrum Akademischer Verlag, Heidelberg, 1996

6. E. Hubble, "A Relation between Distance and Radial Velocity among Extra-Galactic Nebulae". Proceedings of the National Academy of Sciences of the United States of America, vol. 15(3), pp. 168-173, 1929

7. J.C. Pecker and J.P. Vigier, Observational Cosmology. Ed. by A. Hewitt, G. Burbidge, and L.Z. Fang (IAU, Bejing), p. 507, 1987

8. H.C. Arp, Quasars, Redshifts and Controversies. Interstellar media, Berkeley, California, 1987

9. H.C. Arp, G. Burbidge, F. Hoyle, J.V. Narlikar and N.C. Wickramasinghe N.C., "The extragalactic universe: An alternative view". NATURE, vol. 346, p. 801, 1990

10. H.C. Arp, "Der kontinuierliche Kosmos", Mannheimer Forum, Boehringer Mannheim, Ed.by.E.P. Fischer, 1993, p. 113

11. S. Dodelson, Modern Cosmology: Anisotropies and Inhomogeneities in the Universe. Academic Press Inc., 2003

12. H.J. Fahr and M. Heyl, "How are Cosmic Photons Redshifted?", AdAp, vol. 2, no. 1, pp. 1-7, 2017

13. R.V. Pound and J.R. Snider, Physical Review, vol. 140B, p. 788, 1965

14. H.J. Fahr and E.P. Scholz, "Photons in a homogeneous, massive universe", Preprint, 2014 